\title{
Nemesis - a molecular modeling package
}

\author{
Jakub Štěpán ${ }^{1,2^{*}}$, Petr Kulhánek ${ }^{1,2}$, Milan Lenčo ${ }^{3}$, Zora Střelcováa ${ }^{1,2}$, Aleš Křenek ${ }^{3}$, Jaroslav Koča ${ }^{1,2}$ \\ From 8th German Conference on Chemoinformatics: 26 CIC-Workshop \\ Goslar, Germany. 11-13 November 2012
}

Very important part of theoretical simulations is a process of structure preparation, geometry editing and detailed examination of properties. Here we present advanced molecular modeling package called Nemesis [1]. The software is mainly focused on the molecular structure preparation for calculations, taking into account typical user problems. Nemesis is open-source, multi-platform, modular, intuitive and easy to use. The package functionality is exhibited to end-users via projects. Currently, four project types are supported - Sketch Structure, Build Structure, Collaboration, and Trajectory.

The first mentioned, Sketch Structure project offers simple structure drawing in 2D. It is based on limited functionality of the Ketcher editor [2]. More advanced structure building is offered by the Build Structure project. This project is intended for building structures from predefined 3D fragments or single atoms, it offers also tools for typical user tasks as changing of atomic charge, bond order, names, connectivity, or translations, rotations and copying of atoms or atom groups. Prepared structures can be optimized by various force fields with imposition of geometrical restraints. Structure topologies and geometries can be easily altered and/or measured. The package fully supports an import and export of various molecular formats using OpenBabel library [3] and internal frameworks. Several graphical representations can be used to visualize structures and their properties. The Collaboration project extends the Build Structure project by tools necessary for collaborative structure modeling and visualization in group of users. Users can share the same view of modeled structure in real time and structure changes are propagated to all users in real time as well. Finally, the Trajectory project can be used to analyze results from molecular dynamics simulations or geometry optimization and coordinate driving performed by external programs.

The package is written in $\mathrm{C}++$ programming language. Its functionality can be extended via plugins. We are intensively working on extensions focused on RMSD calculation, scripting possibilities, or advanced work with biomolecules containing standard residues. We also continuously add and extend structure visualization models and abilities.

\section{Acknowledgements}

This work was realized in CEITEC - Central European Institute of Technology with research infrastructure supported by the project CZ.1.05/1.1.00/02.0068 financed from European Regional Development Fund (P.K., J.K.). The work has been supported by the Grant Agency of Czech Republic (GD301/09/ H0040) (J.S., Z.S.). The funding provided under the program Center CERIT Scientific Cloud, part of the Operational Program Research and Development for Innovations, reg. no. CZ. 1.05/3.2.00/08.0144 is appreciated (A.K.).

\section{Author details}

${ }^{1}$ CEITEC - Central European Institute of Technology, Masaryk University, 62500 Brno, Czech Republic. ${ }^{2}$ National Centre for Biomolecular Research, Masaryk University, 61137 Brno, Czech Republic. ${ }^{3}$ Institute of Computer Science, Masaryk University, 60200 Brno, Czech Republic.

Published: 22 March 2013

\section{References}

1. Nemesis. [https://lcc.ncbr.muni.cz/whitezone/development/nemesis].

2. Ketcher, GGA software services, Cambridge, USA. [http://www. ggasoftware.com].

3. O'Boyle NM, Banck M, James CA, Morley C, Vandermeersch T, Hutchison GR: J Cheminf 2011, 3:33.

\section{doi:10.1186/1758-2946-5-S1-P12}

Cite this article as: Štěpán et al:: Nemesis - a molecular modeling package. Journal of Cheminformatics 2013 5(Suppl 1):P12.

\footnotetext{
* Correspondence: xstepan3@chemi.muni.cz

'CEITEC - Central European Institute of Technology, Masaryk University,

62500 Brno, Czech Republic

Full list of author information is available at the end of the article
}

C 2013 Štěpán et al.; licensee BioMed Central Ltd. This is an Open Access article distributed under the terms of the Creative Commons 